\title{
Metabolic cardiomyopathy from propionic acidemia precipitating cardiac arrest in a 25-year-old man
}

\author{
Nigel S. Tan MD, Ravi R. Bajaj MD, Chantal Morel MD, Sheldon M. Singh MD
}

Cite as: CMAJ 2018 July 23;190:E883-7. doi: 10.1503/cmaj.180240

A 25-year-old man with a medical history of propionic acidemia was brought to hospital after he collapsed while jogging. He was unresponsive, received immediate bystander cardiopulmonary resuscitation and was defibrillated by paramedics from an initial rhythm of ventricular fibrillation with successful return of spontaneous circulation. He had been diagnosed with propionic acidemia at two years of age when he presented with developmental delay, and metabolic work-up showed the classic profile of 3-OH-propionic and methylcitric acids on analysis of organic acids in urine, low carnitine with increased propionylcarnitine on acylcarnitine profile and increased glycine on quantitative amino acid analysis of plasma. He was found to carry the c.331C > T (p.Arg111*) mutation and the c.1316A > G (p.Tyr439Cys) mutation in the PCCB gene, both of which have been reported previously to be pathogenic. ${ }^{1-3}$ Except for mild developmental delay and learning disabilities, he had no other comorbid medical conditions or previous admissions to hospital in adulthood. He was in good general health, had successfully completed high school and was followed up yearly since childhood at a genetic metabolic clinic. He was prescribed a strict low-protein diet (limited to $40 \mathrm{~g}$ of natural protein daily), as well as supplements including L-carnitine, a multivitamin and a specialized protein substitute (Vitaflo methylmalonic acidemia/ propionic acidemia cooler, $40 \mathrm{~g}$ in total daily). He was not taking any other prescription medications.

At the time of the patient's witnessed cardiac arrest, initial laboratory investigations showed an anion gap metabolic acidosis and lactic acidemia (lactate level $7.9 \mathrm{mM}$ ). His hematologic profile, cardiac biomarkers, serum ammonia level, drug screen and electrocardiograms (ECGs) were normal (including QTc interval $425 \mathrm{~ms}$; unchanged from previous ECGs in clinic). As part of routine follow-up for propionic acidemia, he had been undergoing regular echocardiographic monitoring; his most recent study (conducted three months before his cardiac arrest) showed mild left ventricular systolic dysfunction (left ventricular ejection fraction [LVEF] = 49\%; Figure 1), which was not observed in monitoring that was conducted two years earlier.

Echocardiography conducted after the cardiac arrest quantified the patient's LVEF to be further reduced at $35 \%$. On further

\section{KEY POINTS}

- Metabolic cardiomyopathy is an important diagnostic consideration in a young patient with unexplained ventricular dysfunction or sudden cardiac arrest.

- Propionic acidemia is an uncommon but important cause of metabolic cardiomyopathy because of an enzyme defect that impairs the metabolism of proteins and fats.

- Dietary nonadherence in patients with propionic acidemia can precipitate ventricular dysfunction and malignant arrhythmias, which are potentially reversible.

- Young patients with unexplained cardiomyopathy should be referred to a clinical geneticist for comprehensive evaluation.

discussion, the patient admitted that he had not been restricting his protein intake and had stopped taking supplements altogether. He was treated with intravenous glucose and lipid solutions, which is the standard therapy administered in acutely ill patients with propionic acidemia - to reverse the catabolic state precipitated by the stress of his cardiac arrest and resuscitation. Within 72 hours, he was extubated and the biochemical abnormalities had normalized, with no urine ketones on serial assessments or further arrhythmias on telemetry monitoring. A subsequent coronary angiography was normal, excluding coronary anomalies or obstructive coronary artery disease. Cardiac magnetic resonance imaging (MRI) performed after correction of all metabolic derangements found mildly reduced left ventricular systolic function (LVEF of $51 \%$, normal level $>56 \%$ by MRI), with normal wall motion and no late gadolinium enhancement. There were no other features on imaging to suggest an alternate diagnosis of cardiomyopathy.

Although the patient's ventricular arrhythmia may have been due to a reversible cause, we decided to proceed with insertion of an implantable cardioverter-defibrillator. We based this on the potential for future dietary nonadherence and uncertainty about the natural history of his cardiomyopathy, which might result in a recurrent cardiac arrest. A subcutaneous implantable cardioverter-defibrillator was inserted, given the patient's young age and absence of an indication for pacing. He declined to undergo an exercise stress test to ensure adequate sensing by 
the implanted subcutaneous cardioverter-defibrillator, to confirm that his QT dynamics were normal and to assess for exercise-induced ventricular tachycardia.

During the year after the cardiac arrest, the patient adhered to his diet, remained arrhythmia free and had sustained normalization of his LVEF on serial echocardiograms (Figure 1). Plasma acylcarnitine and urine organic acid profiles measured at a clinic visit immediately after being discharged from the hospital were abnormal (propionylcarnitine level 45.1 [normal 1.07 or more] $\mu \mathrm{mol} / \mathrm{L}$; moderate levels of urine $3-\mathrm{OH}$ propionate acid and a high level of urine methylcitrate acid were detected) with low plasma carnitine and increased glycine on quantitative amino acid analysis. These profiles improved after the patient was adherent to dietary therapy for several months (propionylcarnitine level $23.7 \mu \mathrm{mol} / \mathrm{L}$; small amount of urine methylcitrate acid, with an otherwise normal profile for organic acids in urine). He did not resume jogging but maintained his usual day-to-day activities and was not otherwise medically restricted from exercise.

\section{Discussion}

Sudden cardiac arrest in the young is devastating because of its unexpected nature, frequent occurrence in a public location, and potential loss of productivity and life. The broad differential diag- nosis includes coronary anomalies, heritable arrhythmias, cardiomyopathies and myocarditis. Metabolic cardiomyopathies are an important consideration when evaluating young patients with unexplained ventricular dysfunction (Box 1). ${ }^{4}$ They should be suspected when cardiomyopathy is accompanied by other signs suggestive of a multisystem condition, including distinctive physical appearance and skeletal findings, myopathy, neurologic abnormalities and laboratory abnormalities, in particular during acute stressors that expend energy (e.g., anion gap metabolic acidosis, hypoglycemia, hyperammonemia and transaminitis). These conditions are frequently identified in childhood and present with other systemic features that prompt further investigations. ${ }^{5}$ Inborn errors of metabolism comprise a heterogeneous group of uncommon disorders but have a collective estimated prevalence of one or more in 2500 live births ${ }^{6}$ and are estimated to comprise $15 \%$ of all pediatric cardiomyopathies with a diagnosed cause. ${ }^{7}$

\section{Propionic acidemia}

Propionic acidemia is an autosomal recessive disorder characterized by an enzyme defect impairing protein and fat metabolism (Figure 2). The incidence of propionic acidemia in North America is about 1 in 100000 live births, but in certain populations, such as the Inuit living in Greenland, it can be as high as 1 in $1000 .^{8}$ Mutations of the PCCA and $P C C B$ genes result in dysfunction of

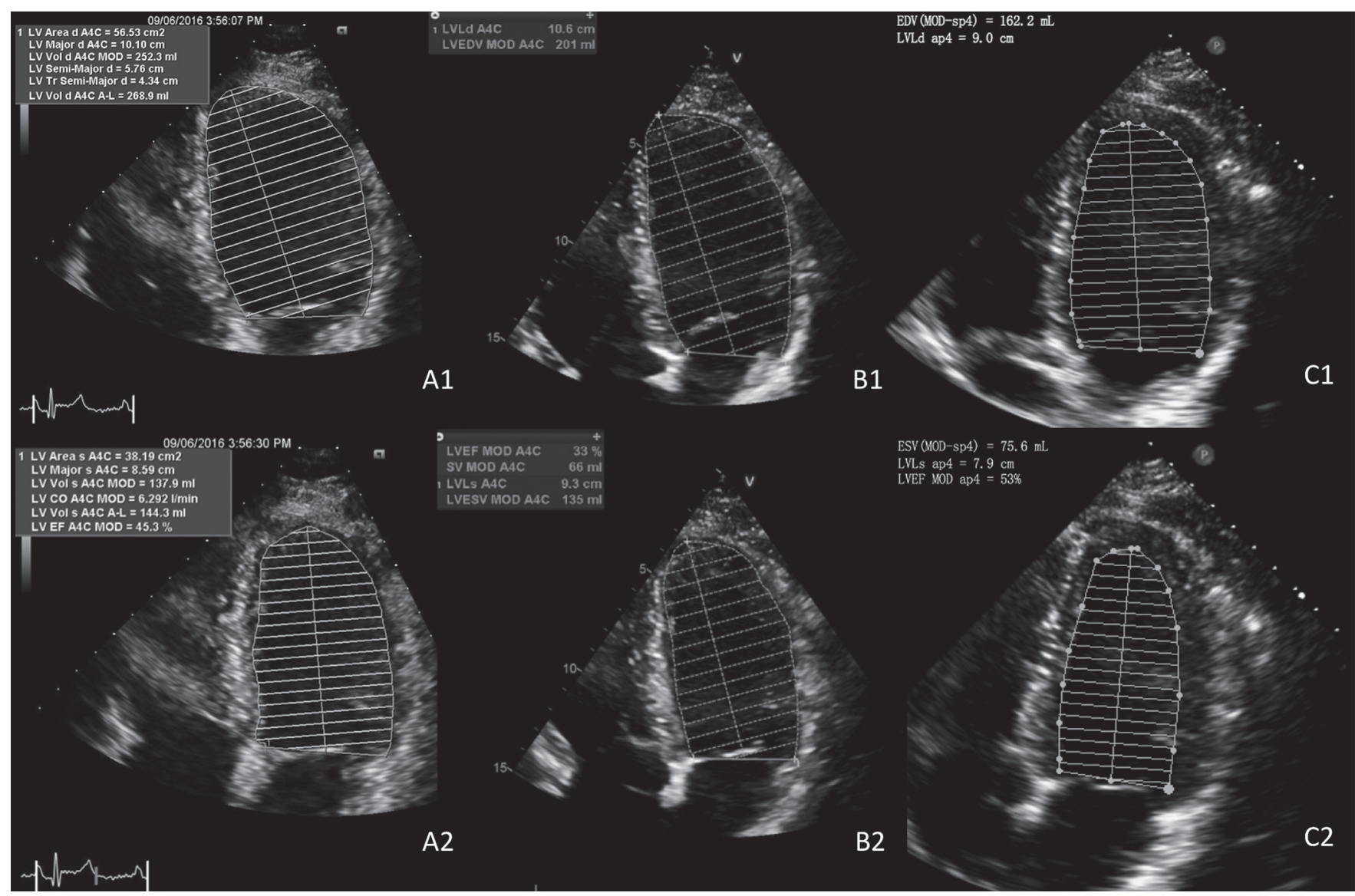

Figure 1: Serial echocardiograms in a 25-year-old man with propionic acidemia several months before admission to hospital after cardiac arrest (A1-2, LVEF 49\%), at time of admission (B1-2, LVEF 35\%) and at several months after discharge while on an appropriate diet (C1-2, LVEF 53\%), showing initial impairment in ventricular function that subsequently improved. Note: EDV = end-diastolic volume, ESV = end-systolic volume, LV = left ventricle, LVEF = left ventricular ejection fraction. 
propionyl coenzyme A carboxylase, which is critical for the metabolism of certain amino acids and lipids.

Propionic acidemia is often diagnosed in the neonatal period, with characteristic patterns for organic acids and acylglycine in urine. In Canada, assessment for propionic acidemia at birth is routine in most provinces and territories as a component of newborn screening programs; however, it is not a component of assessments conducted in the Atlantic provinces. Diagnosis is confirmed with molecular genetic testing for the PCCA and PCCB mutations.

Symptomatic infants present with lethargy, poor feeding, lack of appetite and hypotonia. ${ }^{9}$ However, late-onset presentations also exist, likely owing to partial enzyme activity in milder pheno- types; our patient had a mild form of propionic acidemia, given his relatively high level of function, relatively older age of diagnosis (which predated routine provincial screening) and limited multisystem involvement. A substantial proportion of patients have neurocognitive impairment, seizures, chronic pancreatitis, myopathy and cytopenias. ${ }^{9}$ Cardiac complications are well recognized, with an estimated incidence of cardiomyopathy of up to $23 \%$ in case series. ${ }^{9}$ Isolated cardiac involvement has been reported in teenagers and adults with dilated cardiomyopathy who underwent metabolic screening. ${ }^{10}$ Limited data exist on the incidence of ventricular arrhythmias or cardiac arrest, although prolonged QTc interval is well documented. ${ }^{11}$

\section{Box 1: Inborn errors of metabolism that cause cardiomyopathy}

\author{
Disorders of amino acid and organic acid metabolism \\ - Propionic academia - DCM \\ - Methylmalonic aciduria Malonic academia \\ - $\beta$-Ketothiolase deficiency - DCM \\ - Mevalonic academia \\ - Tyrosinemia-HCM \\ - Oxalosis - HCM, RCM*
}

\section{Disorders of fatty acid metabolism}

Carnitine transport defects

- Systemic primary carnitine deficiency - HCM, DCM

- Muscle carnitine deficiency - HCM, DCM

- Carnitine-palmitoyl transferase type II deficiency

- Carnitine acylcarnitine translocase deficiency

Fatty acid oxidation defects

- Very long-chain acyl-CoA dehydrogenase deficiency - HCM

- Long-chain 3-hydroxyacyl-CoA dehydrogenase deficiency-HCM, DCM

- Short-chain 3-hydroxyacyl-CoA dehydrogenase deficiency

- Multiple acyl-CoA dehydrogenase deficiency (glutaric academic type II) - HCM

\section{Disorders of glycogen metabolism}

- GSD II (Pompe disease, acid $\alpha$-glucosidase/acid maltase) - HCM

- GSD III (Cori disease; debranching enzyme) - HCM

- GSD IV (Anderson disease; branching enzyme) - DCM

- GSD IX (cardiac phosphorylase kinase) - HCM

- PRKAG2 deficiency - HCM with Wolff-Parkinson-White Syndrome

- Danon disease (Pseudo-Pompe disease with normal acid maltase; LAMP2) - HCM

\section{Disorder of glycoprotein metabolism}

- Congenital disorders of glycosylation - HCM

\section{Lysosomal storage disorders}

Disorders of mucopolysaccharide (glycosaminoglycan) metabolism

- MPSI (Hurler, Hurler-Scheie, and Scheie syndromes) - HCM, DCM

- MPS II (Hunter syndrome) - HCM

- MPS III (Sanfilippo syndrome) - HCM

- MPS IV (Morquio syndrome) - HCM

- MPS VI (Maroteaux-Lamy syndrome) - DCM

- MPS VII (Sly syndrome) - HCM
Disorder of glycogen metabolism

- GSD II (Pompe disease, acid $\alpha$-glucosidase/acid maltase) - HCM

- Danon disease (Pseudo-Pompe disease with normal acid maltase; LAMP2) - HCM

Disorders of glycosphingolipid metabolism

- Gaucher disease (glucocerebrosidase) - $\mathrm{HCM}^{*}$

- Fabry disease ( $\alpha$-galactosidase) - $\mathrm{HCM}^{*}$

Disorders of combined ganglioside, mucopolysaccharide, and oligosaccharide metabolism

- GM1 gangliosidosis-HCM, DCM

- GM2 gangliosidosis (Sandhoff disease) - HCM, DCM

Mitochondrial disorders

- Pyruvate dehydrogenase deficiency (Leigh disease) - HCM

- Complex I Deficiency - DCM

- Complex II Deficiency -

- Complex III Deficiency (Histiocytoid cardiomyopathy) - HCM

- Complex IV Deficiency (muscle and Leigh disease forms) - HCM

- Complex V Deficiency - HCM

- MELAS (mitochondrial tRNA mutation) - HCM

- MERFF (mitochondrial tRNA mutation) - HCM, DCM

- Kearns-Sayre syndrome (mitochondrial DNA deletions/ duplications) - HCM

- Barth syndrome (3-methylglutaconic aciduria type II) - HCM, DCM, Mixed

- Sengers syndrome - HCM

\section{Peroxisomal disorder}

- Refsum disease (phytanic acid oxidase) - HCM, DCM

[Note: $\mathrm{CoA}=$ coenzyme A, DCM = dilated cardiomyopathy, $\mathrm{HCM}=$ hypertrophic cardiomyopathy, RCM = restrictive cardiomyopathy.]

${ }^{\star}$ Reported in adults only.

Reprinted from Prog Pediatr Cardiol, Vol 24, GF Cox, Diagnostic approaches to pediatric cardiomyopathy of metabolic genetic etiologies and their relation to

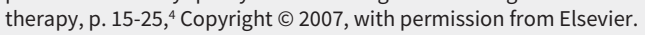




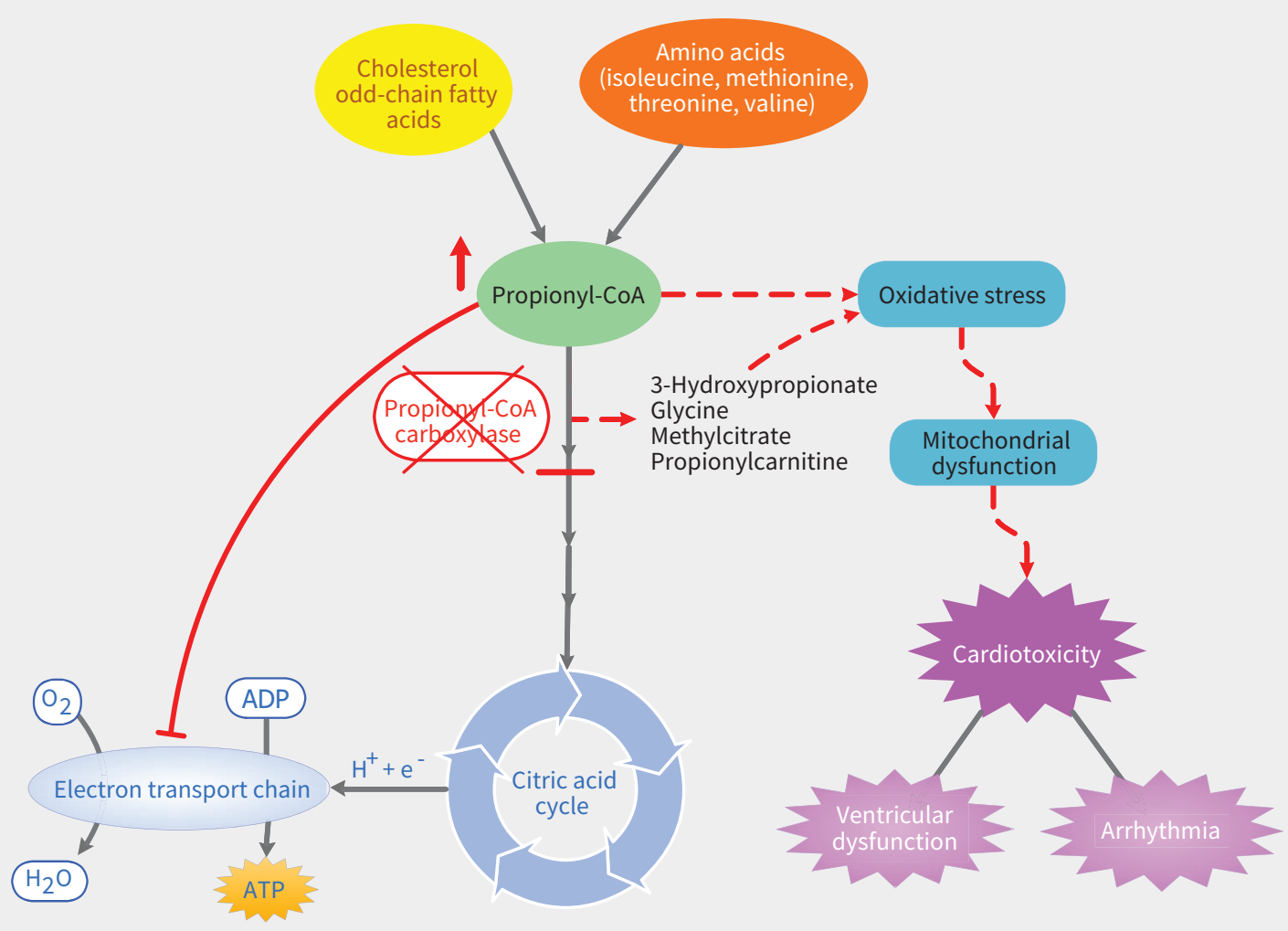

Figure 2: Proposed mechanism of cardiotoxicity in propionic acidemia. A deficiency in propionyl-CoA carboxylase results in accumulation of propionyl$\mathrm{COA}$ and other metabolites. These compounds mediate oxidative damage, compromising the generation of energy-rich adenosine triphosphate (ATP) via oxidative phosphorylation. The resulting cardiotoxicity is manifest in the form of ventricular dysfunction or predisposition to arrhythmogenesis. Note: $\mathrm{ADP}=$ adenosine diphosphate, $\mathrm{CoA}=$ coezyme $\mathrm{A}, \mathrm{e}^{-}=$electron, $\mathrm{H}^{+}=$hydrogen ion, $\mathrm{H}_{2} \mathrm{O}=$ water, $\mathrm{O}_{2}=$ molecular oxygen.

When suspicion exists for a patient affected with a genetic disorder, a referral to a clinical geneticist should be considered. Health care providers should maintain a low threshold for referring a patient with unexplained cardiomyopathy, in particular if there is multisystem involvement or a suggestive family history. Genetic specialists provide comprehensive medical assessments, genetic counselling, genetic testing and ongoing multidisciplinary management when an inherited metabolic disease has been diagnosed.

During acute illness, the mainstay of treatment is to reverse the catabolic state and prevent accumulation of propiogenic compounds, which involves treating the underlying illness, providing calories via intravenous glucose and lipids, and cessation of protein intake for up to 48 hours. ${ }^{9}$ Patients require close monitoring of biochemical parameters including plasma electrolytes, lactate, creatinine, urine ketones, and, ideally, quantitative amino acids and acylcarnitine profile if accurate laboratory analyses can be performed. ${ }^{9}$

Long-term treatment in patients with this condition principally involves adherence to a protein-restricted diet, as well as supplementation with L-carnitine. L-Carnitine binds to propionic acid and aids in excretion of this toxic compound by the kidneys in the form of propionylcarnitines. Biotin, a cofactor for propio- nyl coenzyme A carboxylase, and metronidazole, which reduces intestinal flora that produce propionic acid through fermentation of carbohydrates, also have been used in the treatment for propionic acidemia. ${ }^{9}$

In our patient, we postulate that propionyl coenzyme A, which accumulates in the setting of dietary nonadherence and has been shown to be a mitochondrial toxin, ${ }^{12}$ caused mitochondrial dysfunction and impairment of adenosine triphosphate generation through oxidative phosphorylation, resulting in cardiotoxicity (Figure 2). Other metabolites, including 3-hydroxypropionate, methylcitrate and glycines, may also exert cardiotoxicity at higher concentrations, although this has not been elucidated to date. The degree of organ involvement and disability in propionic acidemia correlates with the frequency of metabolic crises, suggesting that careful acute and long-term management of this condition holds promise to minimize long-term morbidity and mortality. Importantly, a proportion of patients with propionic acidemia will still have persistent cardiomyopathy even with strict dietary and supplement adherence. We suspect that our patient's degree of reversibility related to his mild phenotype, ${ }^{3}$ and it is uncertain to what extent his long-term risk of ventricular dysfunction will be, independent of issues with adherence. Evidence from case reports exists documenting reversal of cardiomy- 
opathy with orthotopic liver transplantation, which is a treatment reserved for select patients with propionic acidemia who have frequent metabolic decompensations and poor quality of life..$^{13}$

Our case highlights the importance of considering metabolic causes when managing patients with idiopathic cardiomyopathy because treatment may be life saving.

\section{References}

1. Brosch S, Rauffeisen A, Baur M, et al. Propionic acidemia and sensorineural hearing loss: Is there a connection at the molecular genetics level? [article in German] HNO 2008 ;56:37-42.

2. Sánchez-Alcudia R, Pérez B, Ugarte $M$, et al. Feasibility of nonsense mutation readthrough as a novel therapeutical approach in propionic acidemia. Hum Mutat 2012;33:973-80.

3. Yorifuji T, Kawai M, Muroi J, et al. Unexpectedly high prevalence of the mild form of propionic acidemia in Japan: presence of a common mutation and possible clinical implications. Hum Genet 2002;111:161-5.

4. Cox GF. Diagnostic approaches to pediatric cardiomyopathy of metabolic genetic etiologies and their relation to therapy. Prog Pediatr Cardiol 2007;24: 15-25.
5. Schwartz ML, Cox GF, Lin AE, et al. Clinical approach to genetic cardiomyopathy in children. Circulation 1996;94:2021-38.

6. Sanderson S, Green A, Preece MA, et al. The incidence of inherited metabolic disorders in the West Midlands, UK. Arch Dis Child 2006;91:896-9.

7. Cox GF. Diagnostic approaches to pediatric cardiomyopathy of metabolic genetic etiologies and their relation to therapy. Prog Pediatr Cardiol 2007;24:15-25.

8. Shchelochkov OA, Carrillo N, Venditti C. Propionic acidemia. In: Adam MP, Ardinger HH, Pagon RA, et al., editors. GeneReviews [Internet]. Seattle (WA): University of Washington;1993-2018.

9. Baumgartner MR, Hörster F, Dionisi-Vici C, et al. Proposed guidelines for the diagnosis and management of methylmalonic and propionic acidemia. Orphanet J Rare Dis 2014;9:130

10. Riemersma M, Hazebroek MR, Helderman-van den Enden ATJM, et al. Propionic acidemia as a cause of adult-onset dilated cardiomyopathy. Eur J Hum Genet 2017;25:1195-201.

11. Baumgartner D, Scholl-Bürgi S, Sass JO, et al. Prolonged QTc intervals and decreased left ventricular contractility in patients with propionic acidemia. $J$ Pediatr 2007;150:192-7-197.e1.

12. Schwab MA, Sauer SW, Okun JG, et al. Secondary mitochondrial dysfunction in propionic aciduria: a pathogenic role for endogenous mitochondrial toxins. Biochem J 2006;398:107-12.

13. Romano S, Valayannopoulos V, Touati G, et al. Cardiomyopathies in propionic aciduria are reversible after liver transplantation. J Pediatr 2010;156:128-34.

\section{Competing interests: None declared.}

This article has been peer reviewed.

The authors have obtained patient consent.

Affiliations: Schulich Heart Program (Tan, Singh), Sunnybrook Health Sciences Centre, Department of Medicine, University of
Toronto; Division of Cardiology, Department of Medicine (Bajaj), North York General Hospital; Fred A. Litwin Family Centre in Genetic Medicine, Department of Medicine (Morel), University of Toronto, Toronto, Ont.

Contributors: Nigel Tan and Sheldon Singh wrote the manuscript. All of the authors reviewed and revised the manuscript for important intellectual content, gave final approval of the version to be published and agreed to be accountable for all aspects of the work.

Correspondence to: Sheldon Singh, Sheldon. singh@sunnybrook.ca 